\title{
Temporal Lobe Anaplastic Astrocytoma
}

National Cancer Institute

\section{Source}

National Cancer Institute. Temporal Lobe Anaplastic Astrocytoma. NCI Thesaurus. Code C156036.

An anaplastic astrocytoma occurring in the temporal lobe. 